\title{
Energy harvesting for IoT road monitoring systems
}

\author{
Rosario Fedele ${ }^{1, *}$, Massimo Merenda ${ }^{1,2}$, Filippo Giammaria \\ Praticò $^{1}$, Riccardo Carotenuto ${ }^{1}$, Francesco Giuseppe Della \\ Corte $^{1,2}$
}

\author{
1. Mediterranea University of Reggio Calabria, Via Graziella - Feo di Vito, \\ Reggio Calabria 89123, Italy \\ 2. HWA S.r.l., Via Reggio Campi II Tr. 135, Reggio Calabria 89126, Italy \\ rosario.fedele@unirc.it
}

\begin{abstract}
Internet of Things (IOT) solutions guarantee the high performance requested by users and authorities in terms of efficiency, sustainability, connectivity, and durability for modern transportation infrastructures, allowing, at the same time, small size, low power consumption, wireless transmission and easily deployable solutions. IoT monitoring systems powered through Energy Harvesting Technologies (EHTs) are often indicated as the most efficient solutions to address these requests because of several advantages (e.g., remote management simplification, independence from electricity grid). In this paper, the most used EHTs in the field of road infrastructures were analyzed and, among them, a photovoltaic standalone system (PVSS) was selected and considered as the power supply unit of an electronic structural health monitoring (SHM) system. In particular, a network of sensor units $(S U s)$, wirelessly connected to one central unit (CU), acting as an innovative road pavement monitoring system solution was taken in account as benchmark. Consequently, the objective of this study is to draw guidelines for the designer that can establish the proper sizing of the PVSS, based on the energy consumption of the SHM system, according to multiple factors, such as typology and number of sensors, frequency of measurements, duty cycle, and days of autonomy.

RÉSUMÉ. Les solutions d'Internet des objets (IdO) garantissent la haute performance demandée par les utilisateurs et les autorités en termes d'efficacité, de durabilité, de connectivité et de durabilité pour les infrastructures de transport modernes, permettant à la fois une petite dimension, une faible consommation d'énergie, une transmission sans fil et des solutions faciles à déployer.

Les systèmes de surveillance d'IdO alimentés par les technologies de récolte d'énergie (EHTs, le sigle de "Energy Harvesting Technologies » en anglais) sont souvent indiqués comme les solutions les plus efficaces pour répondre à ces demandes en raison de plusieurs avantages (par exemple, la simplification de la gestion à distance, l'indépendance du réseau électrique).

Dans cet article, les EHTs les plus souvent utilisées dans le domaine des infrastructures routières ont été analysés et, parmi eux, un système photovoltaïque autonome (PVSS, le sigle
\end{abstract}

Instrumentation, Mesure, Métrologie $-n^{\circ} 4 / 2018,605-623$ 
de "photovoltaic standalone system » en anglais) a été sélectionné et considéré comme l'unité d'alimentation électrique d'un système électronique de contrôle de santé intégré (CSI). En particulier, un réseau d'unités de capteurs (SU, le sigle de "sensor unit » en anglais) connecté sans fil à une unité centrale (CU, le sigle de "central unit » en anglais) qui fonctionne en tant que solution des systèmes de surveillance des chaussées routières innovantes a été pris en compte comme standard. Par conséquent, l'objectif de cette étude est d'élaborer des lignes directrices pour le concepteur en eux permettant d'établir le bon dimensionnement du PVSS, en fonction de la consommation d'énergie du système de CSI anisi que des multiples facteurs, tels que la typologie et le nombre de capteurs, la fréquence des mesures. , cycle de travail et jours d'autonomie.

KEYWORDS: energy harvesting, internet of things, photovoltaic standalone system, road pavement, structural health monitoring system.

MOTS-CLÉS: récolte d'énergie, Internet des objets, système autonome photovoltaïque, chaussée routière, système de contrôle de santé intégré.

DOI:10.3166/I2M.17.605-623 @ 2018 Lavoisier

\section{Introduction}

The efficiency, sustainability, connectivity, and durability of transportation infrastructures are properties strongly requested by users and authorities, and are going to become essential characteristics of the future smart cities. The Intelligent Transportation System (ITS) approach, combining the concept of Internet of Things (IoT) with the Information and Communication Technologies (ICTs) (Pop and Proștean, 2018), aims at redesigning the road infrastructure of the future.

One of the most powerful ITS's tool is represented by the Internet of Things (IoT) solutions, such as the Micro Electro-Mechanical Systems (MEMS) sensor-based monitoring systems (Grace, 2015; Perle et al., 2018). Actually, the IoT Monitoring systems are designed bearing in mind the requests cited above. For this reason, efforts are being made to have more "sustainable" devices, i.e., with a small size, easy to be installed and used, able to carry out monitoring-related activities and wireless data transmission with low power consumption. Possible strategies to achieve this goal include Energy Harvesting Technologies (EHTs) and wireless smart sensor platform equipped with low-power wireless transmitting devices (Saadon and Sideka, 2015; Dhakar, 2017; Praticò et al., 2017; Merenda et al., 2014).

In this section of the paper, recent and relevant examples of EHTs, implemented in road-related applications, are reported together with an overview of the most used low-power technologies for data transmission.

In the remaining sections, an IoT Monitoring system is presented and designed, considering as a power supply unit a photovoltaic standalone system (PVSS). Finally, useful guidelines for designers are provided. 


\subsection{Energy harvesting: road-related applications}

Different approaches were used to gather energy from road infrastructures (including vehicles). Based on (Wang et al., 2018; Duarte and Ferreira, 2016), energy harvesting technologies (EHTs) include systems based on several effects, i.e. photovoltaic, thermal, thermoelectric, geothermal, piezoelectric, electromagnetic, electrostatic. Through these devices, electric energy can be produced, heating or cooling can be carried out, and sensors-based systems can be powered.

Solar-based EHTs for road applications include solar pavements (see e.g., Dezfooli et al., 2017), asphalt solar collectors (e.g., hydronic asphalt pavement a.k.a. HAP, see Pan et al., 2015), and photovoltaic thermal hybrid solar collectors (Xiang et al., 2018). They aim at producing electricity (through photovoltaic systems), or/and heat (through pipes embedded into the pavement in which water flow gathers heat). Photovoltaic (PV) applications have a high level of efficiency and maturity (see Wang et al., 2018; Duarte and Ferreira, 2016). Solar road, solar tree (Hyder et al., 2018), and bus shelters are some of the recent and promising ideas and applications of the PV technology that were proposed in the last years. Compared to traditional PV applications, they were designed (cf. Hyder et al., 2018) to: i) optimize space/land management (especially in urban areas, and open spaces); ii) reduce land carbon footprint compared to the traditional PV solar panel (e.g., solar tree needs less land to be installed); iii) reduce grazing angles of solar irradiation (i.e., the acute angle of incidence of a beam almost parallel to a surface); iv) offer mobile/laptop/electric vehicles charging, street lighting, household/industrial supply, grid support; v) increase the aesthetics of PV, aiming at improving public perception; vi) promote the electric vehicles because of the fact that they have the potentiality to solve one of the main obstacles to their development i.e., the limitation of driving mileage (Youssef, 2018).

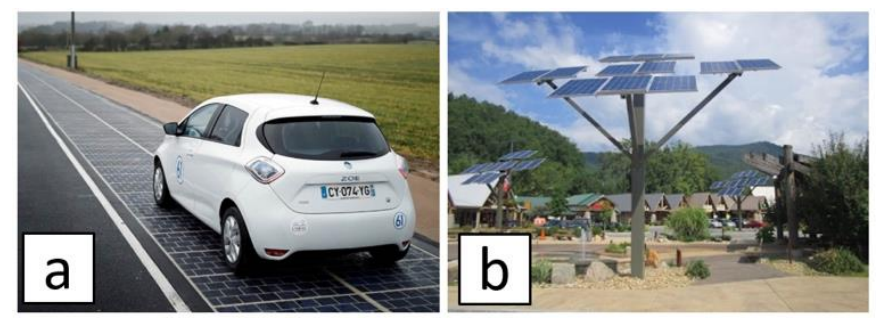

Figure 1. Example of (a) solar road (Silva, 2016), and (b) PV tree (PV-tree)

Collectors in road pavements (a.k.a. Asphalt Solar Collectors, ASC; cf. Duarte and Ferreira, 2016) take advantage from the thermal effect. Their benefits refer to a better control of the pavement temperature (e.g., urban heat island effect reduction; snow melting/deicing on road surface; or air conditioning of buildings (Pan et al., 2015; Xiang et al., 2018). Unfortunately, they need an accurate design (pipe size and distribution, fluid type, and flow rate) and high solar radiation (see Pan et al., 2015; 
Xiang et al., 2018). The main unsolved problems that affect the diffusion of this solar-based EHTs refer to: i) costs (e.g., photovoltaic panel has high investment costs due to the need for inverters and storage batteries; cf. Wang et al., 2018); ii) energy performances (e.g., solar road energy efficiency is about $11 \%$; cf. Xiang et al., 2018); iii) surface performance (e.g., solar pavement have to guarantee suitable roughness, resilience, stiffness, sustainability, etc.; cf. Wang et al., 2018; Dezfooli et al., 2017); iv) construction optimization (e.g., using reinforcing grid to reduce the stress concentration on the pipe of a HAP system; cf. Pan et al., 2015); v) the need for specific designed maintenance processes and long-term performance evaluation; vi) environmental impact (e.g., toxic chemical in the production process, difficulties during recycling and disposal; cf. Wang et al., 2018). Furthermore, they are more suitable where high solar radiation is present. A noteworthy attempt to optimize the energy production of solar roads consists in Photovoltaic thermal hybrid solar collectors (Xiang et al., 2018). The hydronic part of the hybrid system proposed allows decreasing the photovoltaic cell temperatures (by around $24{ }^{\circ} \mathrm{C}$ ) and this increases the electrical efficiencies (by $6 \%$ ) compared to conventional solar roads.

Geothermal EHTs are technologically advanced, but geologically and geographically limited.

Thermoelectric EHTs (i.e., thermoelectric generators, TEGs; cf. Duarte and Ferreira, 2016) are not energetically profitable (i.e., global efficiency 1.6-2.05\%; see Wang et al., 2018; Duarte and Ferreira, 2016) and expensive at present (i.e., equipping one line-mile of road with a typical TEG system and maintain it for all its lifetime, costs 3 times the cost of a typical PV system, and 1.3 times the cost of a solar collector-based system; cf. Wang et al., 2018), Despite this weaknesses, current improvements in materials and structure design are making this technology promising.

Energy from traffic-induced vibrations can be harnessed through piezoelectricbased devices, which are based on piezoelectric, electromagnetic, and electrostatic effects (see Wang et al., 2018). Piezoelectric energy harvesters, where power is generated by mechanical stress, allow stress- or vibration-based energy harvesting, but they need repeated traffic load, and, because of their low energy conversion efficiency, a large number of energy harvesters to gather the energy required from roadways applications. Furthermore, piezoelectric materials undergo structural and electrical degradation due to cycling loading. This calls for proper investigations on long-term durability and service life (see Wang et al., 2018). Besides, significant efforts were made to embed Piezoelectric Energy Harvesters (PEHs) into the road pavement. In fact, it should be considered that: i) embedded devices require tough packaging; ii) vibrations affect their efficiency; iii) their duty cycles should be accorded to the traffic; iv) their design and durability are strictly related to the boundary conditions (e.g., temperature, stress, strain, vibration, etc.); v) standard specifications for their installation into the road, and appropriate management are needed (i.e., implementation of these systems and maintenance; cf. (see Wang et al., 2018; Papagiannakis et al., 2016; Xiong and Wang, 2016; Xu et al., 2017; Hasni et al., 2017; Guo and Lu, 2017; Yang et al., 2018). Despite these problems, these devices allow avoiding the long-distance electricity transmitting, and the energy 
produced by them might be used to power traffic facilities (e.g., signs, or LED traffic lights), or low-power sensors able to get real-time traffic information (e.g., vehicle driving speed and/or load; see Papagiannakis et al., 2016; Xu et al., 2017).

Electromagnetic (EM) systems are easy installable and they are largely applied to bridges (i.e., an EM generator is a velocity-induced transducer that produce energy because of the oscillations of a coil in a magnetic field, induced by the lowfrequency traffic-induced vibrations/oscillations of the bridge; cf. Wang et al., 2018), but they are not able to produce energy from pavement vibrations (Wang et al., 2018; Duarte and Ferreira, 2016).

Electrostatic energy harvesters (i.e., using variable capacitors, induced charges from an external voltage bias or pre-charged) have advantages (e.g., high voltage, low cost, high coupling coefficient, size reduction, etc.) that make them more appropriate for small-scale embedded sensor-based applications (i.e., less $10 \mathrm{~cm}^{3}$ ) than electromagnetic and piezoelectric ones (Wang et al., 2018).

Highway wind turbine on highways medians can produce energy (especially for in series configuration) and reduce accidents (i.e., reduce glare from oncoming vehicles) (Wang et al., 2018).

Vehicle-based EHTs were designed to gather energy from the vehicle vibration. Examples of these devices include piezoelectric system to power wireless sensors on the tire (Lee and Choi, 2014), or to harness car damper energy (Lafarge et al., 2015). Research is growing in this field, aiming at improving electromagnetic generators and hydraulic systems.

Finally, the application of EHTs mostly depends on power output, costeffectiveness, technology readiness level, strengths and weaknesses, and support from government and industry (Wang et al., 2018). It should be noted that none of the EHTs above mentioned have been fully developed, validated, and are today complete and certified products (except for the ASCs, that do not produce electrical energy; cf. Duarte and Ferreira, 2016).

\subsection{Low-power technologies for data transmission}

In order to provide crucial information for the designers of IoT SHM systems concerning the data transmission/interchange among all the devices of this type of systems, different RF-based technologies have been considered in this study. In particular, among all the recent protocols for low-power long-distance data transmission developed in the last years, we considered: i) several Low-Power Wireless (LPW), i.e., ANT, 2.4 and 5 GHz Wi-Fi; ZigBee, RF4CE, Bluetooth Low Energy, and Bluetooth 5, where ANT is a Wireless Personal Network protocol; ii) two Low-Power Wide-Area Networks (LPWAN), i.e. NarrowBand-Internet of Things (NB-IoT) and Long range, low power wireless platform (LoRa).

The main key operating attributes, of these technologies (Mannion, 2017) are frequency band(s), network topology support, throughput (i.e., data transmission rate), range, and coexistence. Each technology makes trade-offs between power 
consumption, bandwidth occupation, and range (Dementyev et al., 2013). Usually, the peak transmit power must be lower than $+21 \mathrm{dBm}$ (decibel-milliwatts, cf. Mannion, 2017), and several factors (e.g., average receive, transmit, and sleep currents, data rate used, time taken for a node to connect to the hub after waking up, the use of sleep between individual Radio frequency, RF, packets, packet size variations, transmitter and receiver distance, and hub parameters) must be considered to determine the actual power consumption of these technologies (Dementyev et al., 2013; Merenda et al., 2014).

Generally, in order to succeed in high-speed data transmission, during the design process, attention must be paid to the following aspects (Mannion, 2017): i) technologies with high power transmission and high sensitivity (i.e., high Signal-toNoise Ratio, SNR) should be used; ii) the operating environment should be carefully analyzed (e.g., ceilings, walls, human bodies affecting the transmission length), instead of using "ideal" conditions; iii) frequency of the RF carrier should be considered; iv) design layout, mechanics, and coding schemes should be adapted to the specific application (Felini et al., 2014; Farris et al., 2017).

ANT (and the most recent ANT+ and ANT BLAZE) is an ultra-LPW protocol and operates in the $2.4 \mathrm{GHz}$ ISM band (Mannion, 2017). Suitable for coin cell powered sensors, allows months or years of battery life. Originally targeted for sports and fitness, recently it is adopted in the home/industrial automation sectors.

Wi-Fi is very efficient for large data transfers using high-speed throughput, but it has high energy consumption (Mannion, 2017). Although improvements have been made to solve this weakness (e.g., Wi-Fi "HaLow" 2017), it is still unsuitable for low power operation (e.g., coin cell). It operates at 2.4 and $5 \mathrm{GHz}$ bands, while the new low-power, and extended range version, i.e. the Wi-Fi "HaLow", works at 90 MHz ISM band (where ISM stands for Industrial, Scientific and Medical Radio).

ZigBee (and ZigBee PRO) is an LPW specification designed for high security mesh networking in industrial/home automation sectors (Mannion, 2017). It operates in the $2.4 \mathrm{GHz}$ ISM band (as well as $784 \mathrm{MHz}$ in China, $868 \mathrm{MHz}$ in Europe, and $915 \mathrm{MHz}$ in the USA and Australia), it has a data rates of 20 kilobits per second, kbps, at $868 \mathrm{MHz}$, and $250 \mathrm{kbps}$ at $2.4 \mathrm{GHz}$.

RF4CE stands for Radio Frequency for Consumer Electronics (Mannion, 2017), and is a customization of $\mathrm{ZigBee}$ for $\mathrm{RF}$ remote control applications (e.g., television set-top boxes). Using RF, it improves interoperability, line-of-sight (straight path between transmitting antenna and receiving antenna), and limited feature drawbacks of infrared (IR) remote control. It is in competition with ZigBee and Bluetooth low energy.

Bluetooth low energy is one of the new wireless interfaces/protocols that have recently emerged to address the needs of the IoT (e.g., micro-amp average current require, or small battery capacities for wearable devices; cf. Mannion, 2017). Its operation frequency of $2.4 \mathrm{GHz}$ is suitable for asynchronous communication, and infrequent exchange of low volume of data. 
Bluetooth 5 increased raw data rate (Mbit/s) by up to $2 \mathrm{x}$, and transmission range by up to $4 \mathrm{x}$ compared to the Bluetooth low energy.

The main difference between the LPW and LPWAN devices concerns the transmission capacity, i.e., the first ones allow transmission for distance less than $150 \mathrm{~m}$ (Mannion, 2017), while the second ones up to $20 \mathrm{~km}$ in rural zones and 1-5 $\mathrm{km}$ in urban zones (Mekki et al., 2018).

Narrowband (NB)-IoT technology (Mekki et al., 2018; Sinha et al., 2017) offers very low latency, high quality of service, reliability, and high transmission range to the IoT markets. It works at 700-900 MHz, in a bandwidth of $200 \mathrm{~Hz}$, with data rate less than $200 \mathrm{kbps}$, and covers from $1 \mathrm{~km}$ (urban) to $10 \mathrm{~km}$ (rural), and has low interference immunity.

Lo-Ra (i.e., Long-Range; c.f. Mekki et al., 2018; Sinha et al., 2017) has advantages in terms of battery lifetime, capacity, and cost lower-cost device, with very long range (high coverage). It is suitable for infrequent communication rate, for local network deployment, for devices that move at high speeds, and for transmitting tiny amounts of data in long range. It operates at ISM bands of $868 \mathrm{MHz}$ in Europe, $915 \mathrm{MHz}$ in North America, and $433 \mathrm{MHz}$ in Asia. Its bandwidths are 125 and 250 $\mathrm{kHz}$, its data rate is less than $50 \mathrm{kbps}$, covers from $5 \mathrm{~km}$ (urban) to $20 \mathrm{~km}$ (rural), and has a high interference immunity.

The following table shows the expected transmission capability of the technologies mentioned above.

Table 1. Range capability of different low-power technologies for data transmission

\begin{tabular}{|c|c|c|}
\hline Technology & Transmission capability $(\mathrm{m})$ & Reference \\
\hline ANT & $<30$ & (Mannion, 2017) \\
\hline $5 \mathrm{GHz}$ Wi-Fi & $<50$ & (Mannion, 2017) \\
\hline ZigBee/RF4CE & $<100$ & (Mannion, 2017) \\
\hline Bluetooth (low energy) & $<30$ & (Mannion, 2017) \\
\hline 2.4 GHz Wi-Fi & $<150$ & (Mannion, 2017) \\
\hline Bluetooth 5 & $200-400$ & (Mannion, 2017) \\
\hline NB-IoT & $<20000$ & (Mekki et al., 2018) \\
\hline LoRa & $<10000$ & (Mekki et al., 2018) \\
\hline
\end{tabular}

\section{Motivations and objectives}

In Section 1, recent and noteworthy examples of Energy Harvesting Technologies (EHTs), used in application related to the road pavement, and lowpower transmitting protocols were analyzed. Among them, the photovoltaic-based 
ones have been pointed out as the most developed and widespread. On the other hand, more "sustainable" and "user-connected" road infrastructures emerged as a crucial feature of the "smart cities" of the future. IoT monitoring solutions equipped with low-power transmitters have the potential to achieve this goal. Based on the above, in the following section a IoT Structural Health Monitoring (SHM) system is presented. In order to minimize energy consumption, this system was designed considering state-of-art extreme low power devices and techniques. For this reason, it was equipped with a low-power transmission system, and powered by a Photovoltaic Standalone System (PVSS). The objective of this study is to draw useful guidelines for the designer that can establish the proper sizing of the PVSS based on the energy consumption of the SHM system, according to multiple factors, such as typology and number of sensors, frequency of measurements, duty cycles of the devices, and days of autonomy of the system.

\section{Road pavement SHM system: characteristics}

In order to monitor the structural health conditions of a road pavement, the action of the vehicular traffic (e.g., vibrations, noise, loads) and the boundary conditions (e.g., temperature, humidity, presence of water in the bottom layers, availability of electricity) should be taken into account. A well-designed monitoring system should be able to carry out a real-time assessment of the overall road conditions, hopefully in a non-destructive, efficient, safe, and sustainable (i.e., save energy, time, and other resources) way (cf. (Praticò et al., 2009). For these reasons, a network of sensor units (SUs), located on the road pavement (in conditions of nondestructivity and safety), powered through a Photovoltaic Standalone System (PVSS, aiming at system sustainability; see Bataineh and Taamneh, 2017), and wirelessly connected to one central unit (CU) was considered as a suitable IoT SHM system. In more detail, each SU contains different type of low-power sensors and a Radio Frequency (RF) transmitter (sustainability and efficiency). In this study, in order to provide guidelines about the main aspects related to the design of the power supply unit of a monitoring system, a system equipped with one SU (Figure 2) was considered. It includes: 1) one photovoltaic panel (PV panel); 2) one recharge circuit; 3) one battery; 4) one sensor unit.

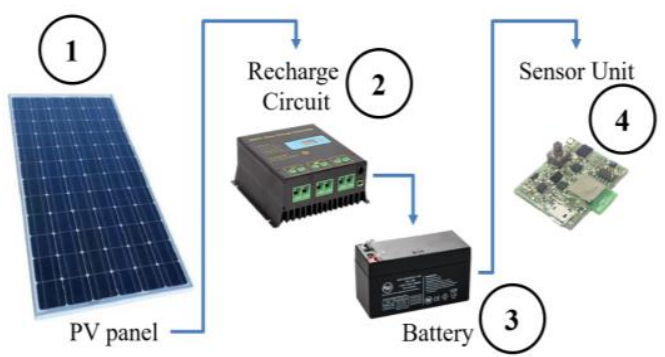

Figure 2. Block diagram of the IoT SHM system 


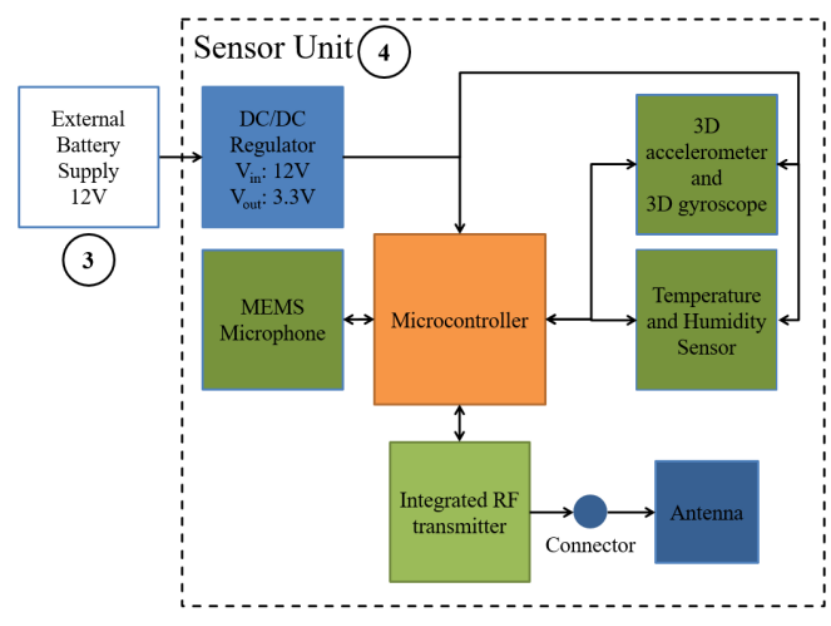

Figure 3. Block diagram of a sensor unit

Figure 3 contains a block diagram that shows all the components of each SU. In more detail, it is possible to see i) the power supply unit, which consists of an external battery and a DC/DC regulator; ii) the sensors chosen to detect the vibroacoustic responses of the pavement to the traffic and important environmental parameters (i.e., temperature and humidity sensors, accelerometer and gyroscope); iii) the microcontroller used to manage the sensors; iv) the RF transmitter unit, which consists of an integrated transmitter and an antenna.

The data gathered through the IoT SHM system above are managed by a software (local or remote) that allows receiving data from RF transmitters, record them on a database, and elaborate and visualize the information derived from the data.

\section{Results and discussions}

\subsection{Road pavement SHM system: power consumption}

In order to derive the energy consumption of the system, it is necessary to define the configuration of the devices used inside the SU (i.e., type, model, etc.) and some important parameters related to their operation, that in fact define a benchmark that will be used during this work. In particular, sampling frequencies and duty cycles have to be specified. For this reason, in Tables 2 and 3, values for these two parameters have been reported. It should be noted that the values of Table 3 were derived based on the content of Table 2. In more detail, the sampling frequencies that are shown in Table 2: i) have been chosen based on a previous study (see Fedele et al., 2017); ii) might allow gathering a sufficient amount of data from which the 
structural conditions of the pavement can be extracted; iii) have been used to calculate the energy consumption of each component of the system. The current consumption of each component of the SU (see Table 3) was derived considering the sensors set chosen (see references in Table 2), a voltage of $3 \mathrm{~V}$, a microcontroller with a clock frequency $f_{c l k}=40 \mathrm{MHz}$, and defining the duty cycles reported in Table 3.

Table 2. Sensor unit (SU) devices, sampling frequencies and power consumptions

\begin{tabular}{|c|c|c|}
\hline Device & Sampling frequency $[\mathrm{kHz}]$ & $\begin{array}{c}\text { Power } \\
\text { consumption }\end{array}$ \\
\hline Accelerometer (STMicroelectronics) & 1 & \multirow{4}{*}{$<2 \mathrm{~mW}$} \\
\hline Gyroscope (STMicroelectronics) & 1 & \\
\hline Microphone (Almeida, 2016) & 44 & \\
\hline Temperature/Humidity sensor (Zito et al., 2010) & 0.1 & \\
\hline Microcontroller (Microchip) & N/A & $\begin{array}{c}150 \mu \mathrm{W} / \mathrm{MHz} \\
\text { in low power } \\
\text { mode }\end{array}$ \\
\hline Low-Power RF Transmitter & N/A & $\begin{array}{l}\text { Based on the } \\
\text { selected } \\
\text { protocol }\end{array}$ \\
\hline
\end{tabular}

Table 3. Duty cycles and current consumption of each component of the sensor unit during the system operation @ fclk=40 MHz

\begin{tabular}{|c|c|c|c|c|c|c|c|}
\hline \multicolumn{2}{|c|}{} & \multicolumn{5}{|c|}{ Duty cycle of operation (\%) } & \\
\hline \multicolumn{2}{|c|}{ Operational condition $^{1}$} & 0 & 1 & 2 & 3 & \\
\hline \multirow{2}{*}{ Device } & State & \multicolumn{5}{|l|}{} & $\mathrm{CC}^{5}$ \\
\hline \multirow{2}{*}{$\mathrm{M}^{2}$} & Sleep & 99 & 99 & 99 & 99 & 0.01 \\
\cline { 2 - 8 } & Run & 1 & 1 & 1 & 1 & 15 \\
\hline \multirow{2}{*}{$\mathrm{S}^{3}$} & Sleep & 90 & 60 & 30 & 0 & 0.1 \\
\cline { 2 - 8 } & Run & 10 & 40 & 70 & 100 & 5 \\
\hline \multirow{2}{*}{$\mathrm{RF} / \mathrm{Wi}-\mathrm{Fi}^{4}$} & Sleep & 90 & 95 & 97.5 & 99 & 0.1 \\
\cline { 2 - 8 } & Run & 10 & 5 & 2.5 & 1 & 150 \\
\hline
\end{tabular}

Notes: 1. Operational conditions (see Figure 7). 2. Microcontroller. 3. Sensors. 4. Transmitter system/protocol. 5. Current consumption at $3 \mathrm{~V}(\mathrm{~mA})$. 
Based on Tables 3, it is possible to calculate the average daily power consumption of the IoT SHM, for one $\mathrm{SU}(=\mathrm{M}+\mathrm{S}+\mathrm{RF} / \mathrm{Wi}-\mathrm{Fi})$ : i) for each device (e.g., M), the current consumption (A) is derived (sleep + run); ii) the average current consumption (A) is then derived; iii) the average power consumption is calculated $(\mathrm{A} \cdot \mathrm{V}=\mathrm{W})$; iv) the 24h-related energy consumption is derived $(\mathrm{W} \cdot \mathrm{h})$; v) this latter represents the energy needed in a day (about $380 \mathrm{mWh} /$ day).

This result will be used in the following section to properly size the power supply system.

\subsection{Power supply system: characterization and sizing}

The characterization and sizing of the power supply system were based on the energy consumption of the system with the operating condition specified in the benchmark (as defined in the previous section), and under the hypothesis of installing the SHM system on a road in Reggio Calabria (Italy), herein called "reference road". This road was selected as an example of road that needs to be monitored because: 1) it is located very close to a small river (creek) and for this reason, it is subjected to high hydrogeological risk; 2) section location and characteristics easily allow gathering information about traffic spectrum and solar radiation. Indeed, in order to define the characteristics of the power supply system (i.e., the dimensions, peak power, etc.), the solar radiation (i.e., irradiation) is required.

Different papers and tools are available online that allow estimating the solar radiation, and sizing photovoltaic (PV) grid-connected and off-grid systems (e.g., Chabane et al., 2018; ENEA; JRC Europe; SODA; Oregon Embedded). In this study, the tool provided by JRC Europe was used, to draw Figure 6, which shows the annual solar irradiation on the reference road (see Figure 4).

In particular, the highest values of irradiation refer to the optimal inclination $\left(I_{\text {opt }}\right)$ of the solar panel (i.e., the acute angle between a horizontal plane and an inclined plane facing south). The $I_{\text {opt }}$ allows gathering the maximum amount of solar energy because it varies during the year. From the $I_{\text {opt }}$ curve, the average optimal inclination was calculated (i.e., $36^{\circ}$ ), and the irradiation $H(36)$ was determined. The annual averages (av) values are the following: Irradiation on horizontal plane $H_{h_{-} a v}=4889$ $\mathrm{Wh} / \mathrm{m}^{2} /$ day; Direct normal irradiation $(\mathrm{DNI})=5306 \mathrm{Wh} / \mathrm{m}^{2} /$ day; Irradiation on optimally inclined plane $H_{\text {optav }}=5523 \mathrm{Wh} / \mathrm{m}^{2} / \mathrm{day}$; Irradiation on plane at the average optimal inclination angle of $36^{\circ}, H(36)_{a v}=5508 \mathrm{Wh} / \mathrm{m}^{2} /$ day. Because of the fact that, $H(36)_{a v}$ is very close to the maximum irradiation $\left(H_{\text {opt_av }}\right)$, it is possible to assume this value as the input parameter of the PV panel (i.e., the panel inclination was assumed as $36^{\circ}$ ). 


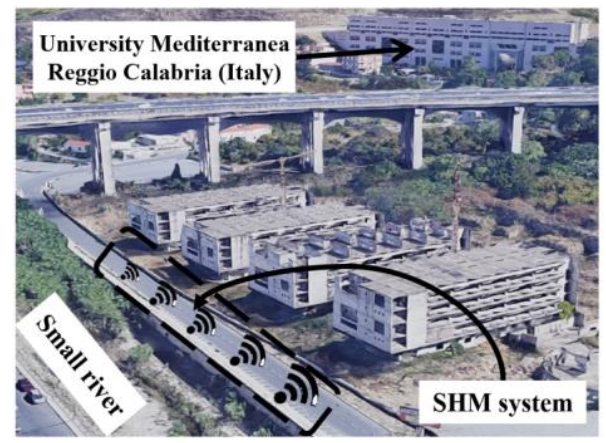

Figure 4. Road reference beside a small river in Reggio Calabria (Italy) where we suppose to install the SHM system

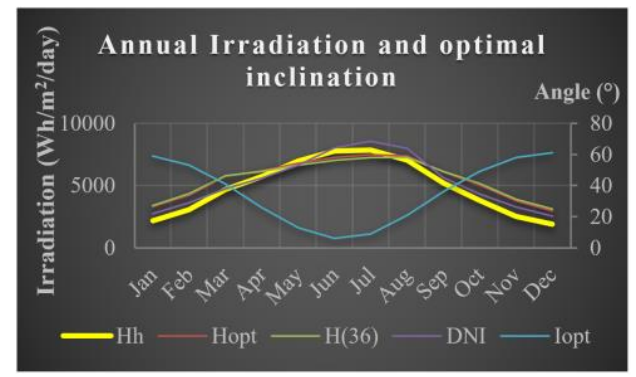

Figure 5. Annual trend of the daily irradiation on the reference road obtained for the optimal inclination (angle) of the panel. Symbols. Hh: Irradiation on horizontal plane; Hopt: Irradiation on optimally inclined plane; H(36): Irradiation on plane at the average optimal inclination angle: $36^{\circ}$; DNI: Direct normal irradiation; Iopt:

Optimal inclination $\left(^{\circ}\right)$

The power supply unit (PV panel and battery) was calculated using the following equations (Kazem et al., 2013):

$$
A_{P V}=\frac{E_{P V}}{H \times \eta_{P V} \times \eta_{\text {wire }}},
$$

where $A_{P V}$ is the area of the PV panel $\left(\mathrm{m}^{2}\right) ; E_{P V}$ is the energy produced by the PV panel in one day (Wh/day); $H$ stands for the annual irradiation $\left(\mathrm{Wh} / \mathrm{m}^{2} /\right.$ day $) ; \eta_{P V}$ is the efficiency of the PV panel (\%; as reported into the manufacturer data sheet; e.g., $14 \%) ; \eta_{\text {wire }}$ is the efficiency of the wire used into the system (\%; this value is a function of quality and length of the wires used). 
In this study, a battery was included in the power supply unit because of the fact that this allows increasing the reliability of the system. In more detail, if the sizing of the battery is conveniently carried out, the system will be able to perform its work even in the case of the failure of the PV panel. This will permit, to the decision maker (e.g., authority or the company that are responsible of the system maintenance), to define and make the proper intervention to solve the failure and rehabilitate the system.

The following equation was used to size the battery:

$$
C_{B}=\frac{E_{L} \times n}{D O D \times \eta_{B} \times V_{B}},
$$

where $C_{B}$ is the capacity of the battery $(\mathrm{Ah}) ; E_{L}$ is the daily energy load (or daily energy consumption, i.e., the energy required by the $\mathrm{SU}$ of the system as a function of the operational condition in a day); $n$ represents the autonomy of the system provided by the battery (day); DOD stands for the maximum depth of discharge of the battery (\%; usually, greater than $20 \%$, e.g., $50 \%$; cf. Affordable Solar); $\eta_{B}$ is the efficiency of the battery $(\%) ; V_{B}$ is the nominal voltage of the battery $(\mathrm{V})$.

\subsection{Examples and guidelines}

In this section, useful curves and guidelines for designers are reported. In the previous section, the average solar radiation $\left(5508 \mathrm{Wh} / \mathrm{m}^{2} /\right.$ day $)$ for the reference road, and the energy load of system $(380 \mathrm{mWh} /$ day $=0.380 \mathrm{Wh} /$ day $)$, in the benchmark condition 0 (see Tables 2 and 3 ) and equipped with one SU (\#SU = 1), were calculated. In order to compensate for the losses of the monitoring system, the energy load of the system was assumed as $1 \mathrm{Wh} /$ day. This value was used into the following examples. The first set of curves (Figure 6) concerns the sizing of the power unit supply (i.e., the PV panel and the battery) of the IoT SHM monitoring system (at the benchmark condition 0 , and \#SU = 1). Appling Eq. (1), using as input: $E_{P V}=1 \mathrm{Wh} /$ day, $\mathrm{H}=5508 \mathrm{Wh} / \mathrm{m}^{2} /$ day, $\eta_{P V}=14 \%$, and $\eta_{\text {wire }}=85 \%$, the area of the panel required by the system equipped with $1 \mathrm{SU}$ was $0.0015 \mathrm{~m}^{2}$ (e.g., a panel with an area of $10 \times 15 \mathrm{~cm}^{2}$ ). After that, using the Eq. (2) and a PV panel with an area equal to $0.0015 \mathrm{~m}^{2}$, the following curves were drawn (Figure 6). These curves show how the capacity of the battery $\left(C_{B}\right)$ is influenced by the days of autonomy $(n)$, and the voltage of the battery $\left(V_{B}\right)$ when the other parameters are fixed (i.e., $E_{L}=1$ $\mathrm{Wh} /$ day; $\left.D O D=50 \% ; \eta_{B}=80 \%\right)$. 


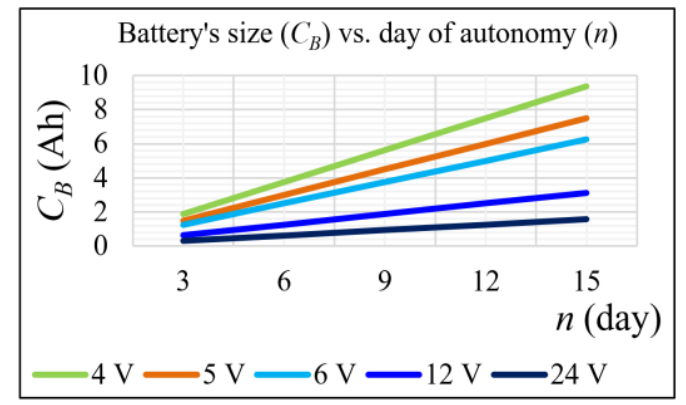

Figure 6. Capacity of the battery $\left(C_{B}\right)$ as a function of the day of autonomy (n), and battery voltage

If the benchmark conditions or the number of SU change, the energy consumption of the monitoring system changes as well. For this reason, in the next example, the energy consumption of the system equipped with $1 \mathrm{SU}$ was estimated changing the benchmark conditions as in Table 3. Figure 7 shows: i) the operational conditions $0,1,2,3$ (x-axis, cf. Table 3); ii) the run time percentage, per given device ( $R \_M, R \_S, R \_R F$, respectively, cf. y-axis, left); iii) the energy load per day needed per given operational condition $\left(\mathrm{E}_{\mathrm{L}}, \mathrm{y}\right.$-axis, right).

The results shown in Figure 7 demonstrate that it is possible to optimize the system, reducing the energy consumption and improving the performances of the system (in terms of duty cycle) at the same time.

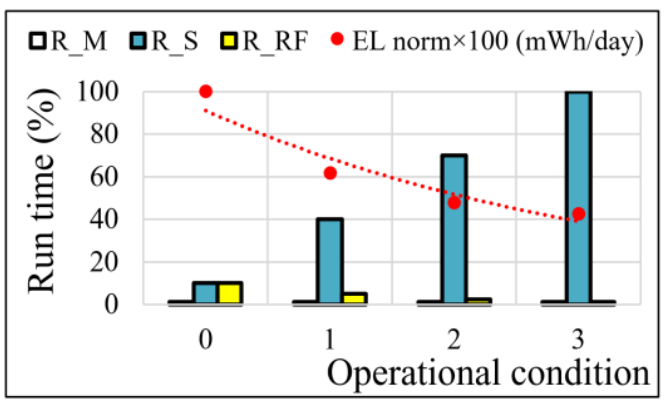

Figure 7. Energy consumption of the system $\left(E_{L}\right)$ equipped with $1 S U$ (containing the microcontroller $M$, the sensors $S$, and the data transmitter $R F)$ as a function of the duty cycle

Figure 8 shows: i) the energy consumption per day $\left(\mathrm{E}_{\mathrm{L}}\right)$ of the IoT SHM system (at the benchmark condition 0 defined in Tables 2 and 3 ) as a function of the number of the SU used (\#SU); ii) the area of a PV panel (APV) that should be used to power the IoT system equipped with a network of SUs; iii) the capacity of the battery $\left(C_{B}\right.$; 
in Ah divided by 10 to be plotted in this graph) at different voltages (i.e., 4, 5, 6, 12, $24 \mathrm{~V}$ ) that should be used to power the monitoring system equipped with a network of SUs in order to ensure 15 days of autonomy.

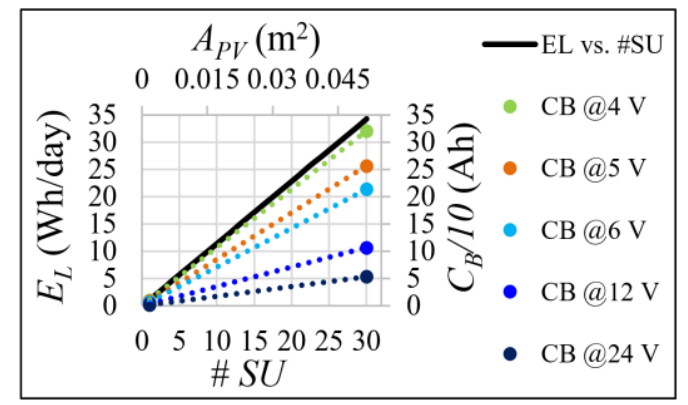

Figure 8. Energy load of the system per day $\left(E_{L}\right)$, battery capacity $\left(C_{B}\right)$ at different voltages $(V)$, and area of the PV panel $\left(A_{P V}\right)$ as a function of the number of Sensor Unit (\#SU)

Finally, considering that a real IoT monitoring system consists in a network of SUs wireless connected to a central unit (CU), the design of all the system components should be carefully carried out. For this reason, the following procedure was drawn: 1) Consider the number of SUs per linear meter (e.g., 0.1) controlled by one CU. 2) Consider a standalone power supply that consists of the following components: i) Photovoltaic modules; ii) Batteries; iii) DC/DC regulator (where DC stands for direct current). 3) Define sampling frequencies, and duty cycles of the devices of the IoT SHM System. Note that, in order to define these parameters, a trade-off between real-time monitoring and the energy consumption is needed. 4) Calculate the energy consumption of the devices above as a function of sampling frequency and duty cycles defined in the previous point. 5) Define a RF transmission system and the relative energy consumption (derived from the datasheet of the devices used). 6) Calculate the maximum irradiation (best angle and orientation) in the place where the IoT SHM system has to be installed. 7) Calculate the dimension of a PV panel (or more than one) that is able to produce the energy daily required by the IoT SHM System. 8) Determinate the characteristics of a battery that allows gathering enough energy to power the system even in case of failure or reduction of energy production by the PV panel.

\section{Conclusion}

Smart city and Intelligent Transportation System (ITS) need low-consuming, efficient, durable, and innovative solutions to make "smarter" the current road infrastructures. Future transportation assets will be able to ensure high performances and, at the same time, share useful piece of information to users and authorities. In order to achieve this objective, IoT Monitoring system equipped with low-power 
data transmitters are a possible solution. For this reason, in this paper, an innovative IoT Structural Health Monitoring (SHM) system was presented and sized. In order to minimize energy consumption, this system was equipped with a low-power transmission system and powered by a Photovoltaic Standalone System (PVSS).

ANT, Wi-Fi, ZigBee/RF4CE, Bluetooth, NB-Iot, and LoRa protocols were taken into account as possible data transmission protocols of the proposed system. Their main performances were reported and compared. As power supply unit of the IoT SHM system, a Photovoltaic Standalone System (PVSS) was considered and different solutions were presented and compared to optimize the energy management.

The study aims at providing useful guidelines for the designer to make easier the implementation of IoT solutions in road infrastructure, in particular regarding the sizing of the power supply system using PVSS systems as a function of the load conditions that change in accordance to the performance requested to the IoT solutions. Results show different possibilities offered by the customizable IoT SHM system proposed, and provide several possible configurations according to multiple factors, such as typology and number of sensors, frequency of measurements, duty cycles, and autonomy of the system provided by a battery.

\section{References}

Affordable Solar. Available: http://www.affordable-solar.com/learning-center/solarbasics/off-grid-system-sizing/

Almeida C. (2016). Integração de sensores inteligentes para a supervisão remota de subestações secundárias de distribuição de energia eléctrica, M.S. thesis, Dep. Phys., Univ. Sc. \& Tech., Coimbra, PT, 2016.

Bataineh K., Taamneh Y. (2017). Performance analysis of stand-alone solar dish Stirling system for electricity generation. International Journal of Heat and Technology IJHT, Vol. 35, No. 1, pp. 498-508. https://doi.org/10.18280/ijht.350306

Chabane F., Laznek I., Bensahal D. (2018) Prediction of global solar radiation on the horizontal area with the effect of relative humidity part: I. Italian Journal of Engineering Science: Tecnica Italiana IJES, Vol. 61+1, No. 2, pp. 115-118. https://doi.org/10.18280/ijes.620209

Dementyev S., Taylor H. S., Smith J. (2013). Power consumption analysis of bluetooth low energy, ZigBee and ANT sensor nodes in a cyclic sleep scenario. Presented at IEEEIWS13 Annual Meeting. https://doi.org/10.1109/IEEE-IWS.2013.6616827

Dezfooli A. S., Nejad F. M., Zakeri H., Kazemifard S. (2017). Solar pavement: A new emerging technology. Solar En., Vol. 149, pp. 272-284. https://doi.org/10.1016/j.solener.2017.04.016

Dhakar L. (2017). Triboelectric devices for power generation and self-powered sensing applications. https://books.google.it

Duarte F., Ferreira A. (2016). Energy harvesting on road pavements: State of the art. Presented at ICE-Energy16 Annual Meeting. https://doi.org/10.1680/jener.15.00005 
ENEA. Available: http://www.solaritaly.enea.it/CalcRggmmIncl/Calcola1.php

Farris I., Pizzi S., Merenda M., Molinaro A., Carotenuto R., Iera A. (2017). 6lo-RFID: A framework for full integration of smart UHF RFID tags into the internet of things. IEEE Netw, Vol. 31, No. 5, pp. 66-73. https://doi.org/10.1109/MNET.2017.1600269

Fedele R., Praticò F. G., Carotenuto R., Della Corte F. G. (2017). Sensing road pavement health status through acoustic signals analysis. Presented at PRIME17 Annual Meeting. https://doi.org/10.1109/PRIME.2017.7974133

Felini C., Merenda M., Della Corte F. G. (2014). Dynamic impedance matching network for RF energy harvesting systems. Presented at RFID-TA14 Annual Meeting. https://doi.org/10.1109/RFID-TA.2014.6934206

Grace R. (2015). Sensors to support the IoT for infrastructure monitoring: Technology and applications for smart transport/smart buildings. Presented at MEPTEC-IoT15 Annual Meeting. http://www.meptec.org/Resources/15\%20-\%20Grace.pdf

Guo L., Lu Q. (2017). Modeling a new energy harvesting pavement system with experimental verification. Appl En., Vol. 208, pp. 1071-1082. https://doi.org/10.1016/j.apenergy.2017.09.045

Hasni H., Alavi A. H., Chatt K., Lajnef N. (2017). A self-powered surface sensing approach for detection of bottom-up cracking in asphalt concrete pavements: Theoretical/numerical modelling. Constr \& Build Mat., Vol. 144, pp. 728-746. https://doi.org/10.1016/j.conbuildmat.2017.03.197

Hyder F., Sudhakar K., Mamat R. (2018). Solar PV tree design: A review. Renew \& Sust En Rev., Vol. 82, pp. 1079-1096. https://doi.org/10.1016/j.rser.2017.09.025

JRC Europe. Available: http://re.jrc.ec.europa.eu/pvgis/apps4/pvest.php?lang=it\&map=europe\#

Kazem H. A., Khatib T., Sopian K. (2013). Sizing of a standalone photovoltaic/battery system at minimum cost for remote housing electrification in Sohar, Oman. En \& Build., Vol. 61, pp. 108-115. https://doi.org/10.1016/j.enbuild.2013.02.011

Lafarge B., Delebarre C., Grondel S., Curea O., Hacala A. (2015). Analysis and optimization of a piezoelectric harvester on a car damper. Presented at ICU15 Annual Meeting. https://doi.org/10.1016/j.phpro.2015.08.202

Lee J., Choi B. (2014). Development of a piezoelectric energy harvesting system for implementing wireless sensors on the tires. En Conv \& Man. Vol. 78, pp. 32-38. https://doi.org/10.1016/j.enconman.2013.09.054

Mannion P. (2017). Comparing low-power wireless technologies (Part 1). Digi-Key's North American Editors. Available: https://www.digikey.it/en/articles/techzone/2017/oct/comparing-low-power-wirelesstechnologies

Mekki K., Bajic E, Chaxel F., Meyer F. (2018). A comparative study of LPWAN technologies for large-scale IoT deployment. https://doi.org/10.1016/j.icte.2017.12.005

Merenda M., Farris I., Felini C., Militano L., Spinella S. C., Della Corte F. G., Iera A. (2014). Performance assessment of an enhanced RFID sensor tag for long-run sensing applications. 13th IEEE SENSORS Conference. https://doi.org/10.1109/ICSENS.2014.6985105 
Merenda M., Felini C., Della Corte F. G. (2014). An autonomous and energy efficient smart sensor platform. 13th IEEE SENSORS Conference, pp. 1208-1211. https://doi.org/10.1109/ICSENS.2014.6985226

Microchip. Datasheet device: ATSAMD20E17. Available: http://www.microchip.com

Oregon Embedded. Available: http://www.oregonembedded.com/batterycalc.htm

Pan P., Wu S., Xiao Y., Liu G. (2015). A review on hydronic asphalt pavement for energy harvesting and snow melting. Renew \& Sust En Rev., Vol. 48, pp. 624-634. https://doi.org/10.1016/j.rser.2015.04.029

Papagiannakis A. T., Dessouky S., Montoya A., Roshani H. (2016). Energy harvesting from roadways. Presented at SEIT16 Annual Meeting. https://doi.org/10.1016/j.procs.2016.04.164

Perles A., Pérez-Marín E., Mercado R., Segrelles J. D., Blanquer I., Zarzo M., Garcia-Diego F. J. (2018). An energy-efficient internet of things (IoT) architecture for preventive conservation of cultural heritage. Fut. Gen. Com. Sy., Vol. 81, pp. 566-581. https://doi.org/10.1016/j.future.2017.06.030

Pop M. D., Proștean O. (2018). A comparison between smart city approaches in road traffic management. $\mathrm{Pr} \quad \mathrm{Soc} \& \mathrm{Beh} \quad \mathrm{Sc}$., Vol. 238, pp. 29-36. https://doi.org/10.1016/j.sbspro.2018.03.004

Praticò F. G., Della Corte F. G., Merenda M. (2017). Self-powered sensors for road pavements. Presented at CEW16 Annual Meeting. https://doi.org/10.1201/9781315643274-151

Praticò F. G., Moro A., Ammendola R. (2009). Factors affecting variance and bias of nonnuclear density gauges for PEM and DGFC. The Baltic J Road \& Brid. Eng., Vol. 4, No. 3, pp. 99-107. https://doi.org/10.3846/1822-427X.2009.4.99-107

PV-tree. http://bucket.sunshineworks.com/images/solar-panel-mounts/pv-tree/pv-tree-1r.jpg

Saadon S., Sideka O. (2015). Micro-electro-mechanical system (MEMS)-based piezoelectric energy harvester for ambient vibrations. Proc Soc \& Beh Sc., Vol. 195, pp. 2353-2362. https://doi.org/10.1016/j.sbspro.2015.06.198

Silva D. (2016). World's first solar road opens in Normandy, France. NBC NEWS. Available: https://www.nbcnews.com/science/science-news/world-s-first-solar-road-opensnormandy-france-n699351

Sinha R. S., Wei Y., Hwang S. H. (2017). A survey on LPWA technology: LoRa and NB-IoT. ICT Expr., Vol. 3, No. 1, pp. 14-21. https://doi.org/10.1016/j.icte.2017.03.004

SODA. Available: http://www.soda-is.com/eng/services/services_radiation_free_eng.php

STMicroelectronics. Datasheet device: LSM6DS3. Available: http://www.st.com

Wang H., Jasim A., Chena X. (2018). Energy harvesting technologies in roadway and bridge for different applications - A comprehensive review. Appl En., Vol. 212, pp. 1083-1094. https://doi.org/10.1016/j.apenergy.2017.12.125

Xiang B., Cao X., Yuan Y., Suna L., Wu H., Haghighat F. (2018). A novel hybrid energy system combined with solar-road and soil-regenerator: Dynamic model and operational performance. En Conv \& Man., Vol. 156, pp. 376-387. https://doi.org/10.1016/j.enconman.2017.11.066 
Xiong H., Wang L. (2016). Piezoelectric energy harvester for public roadway: On-site installation and evaluation. Appl En., Vol. 174, pp. 101-107. https://doi.org/10.1016/j.apenergy.2016.04.031

Xu X., Cao D., Yang H., He M. (2017). Application of piezoelectric transducer in energy harvesting in pavement. Int J Pav Res \& Tech. https://doi.org/10.1016/j.ijprt.2017.09.011

Yang H., Wang L., Zhou B., Wei Y., Zhao Q. (2018). A preliminary study on the highway piezoelectric power supply system. Int J Pav Res \& Tech., Vol. 11, pp. 168-175. https://doi.org/10.1016/j.ijprt.2017.08.006

Youssef A. M. (2018). Operations of electric vehicle traction system. Mathematical Modelling of Engineering Problems, Vol. 5, No. 2, pp. 51-57. https://doi.org/10.18280/mmep.050201

Zito F., Aquilino F., Fragomeni L., Merenda M., Della Corte F. G. (2010) CMOS wireless temperature sensor with integrated radiating element. Sensors and Actuators, A: Physical, Vol. 158, No. 2, pp. 169-175. https://doi.org/10.1016/j.sna.2009.12.014 
